Supplement of Hydrol. Earth Syst. Sci., 24, 3097-3109, 2020

https://doi.org/10.5194/hess-24-3097-2020-supplement

(c) Author(s) 2020. This work is distributed under

the Creative Commons Attribution 4.0 License.

(c) (1)

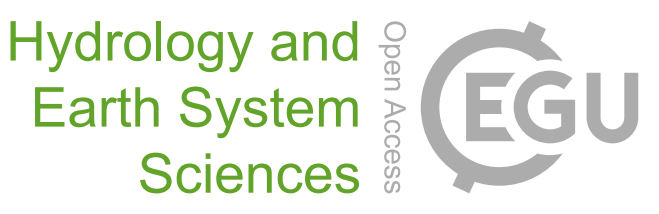

Supplement of

\title{
Interpretation of multi-scale permeability data through an information theory perspective
}

Aronne Dell'Oca et al.

Correspondence to: Aronne Dell'Oca (aronne.delloca@polimi.it)

The copyright of individual parts of the supplement might differ from the CC BY 4.0 License. 


\section{Supplementary Material}

List of Figures included as Supplementary Material:

- Figure S1: Shannon entropy and normalized mutual information associated with data related to the Berea and Topopah samples as a function of as a function of $(i)$ the number of bins (i.e., we consider a number of 50, 75, 100, and 125 bins for the discretization of the range of data variability) and (ii) the size of the kernel bandwidth (which is varied within the range 0.1 - 0.4) employed in the KDE routine.

- Figure S2: Information partitioning of the multivariate mutual information associated with data related to the Berea sample, as a function of $(i)$ the number of bins (i.e., we consider a number of 50,75,100, and 125 bins for the discretization of the range of data variability) and (ii) the size of the kernel bandwidth (which is varied within the range $0.1-0.4$ ) employed in the KDE routine.

- Figure S3: Information partitioning of the multivariate mutual information associated with data related to the Topopah sample, as a function of $(i)$ the number of bins (i.e., we consider a number of $50,75,100$, and 125 bins for the discretization of the range of data variability) and (ii) the size of the kernel bandwidth (which is varied within the range $0.1-0.4$ ) employed in the KDE routine. 

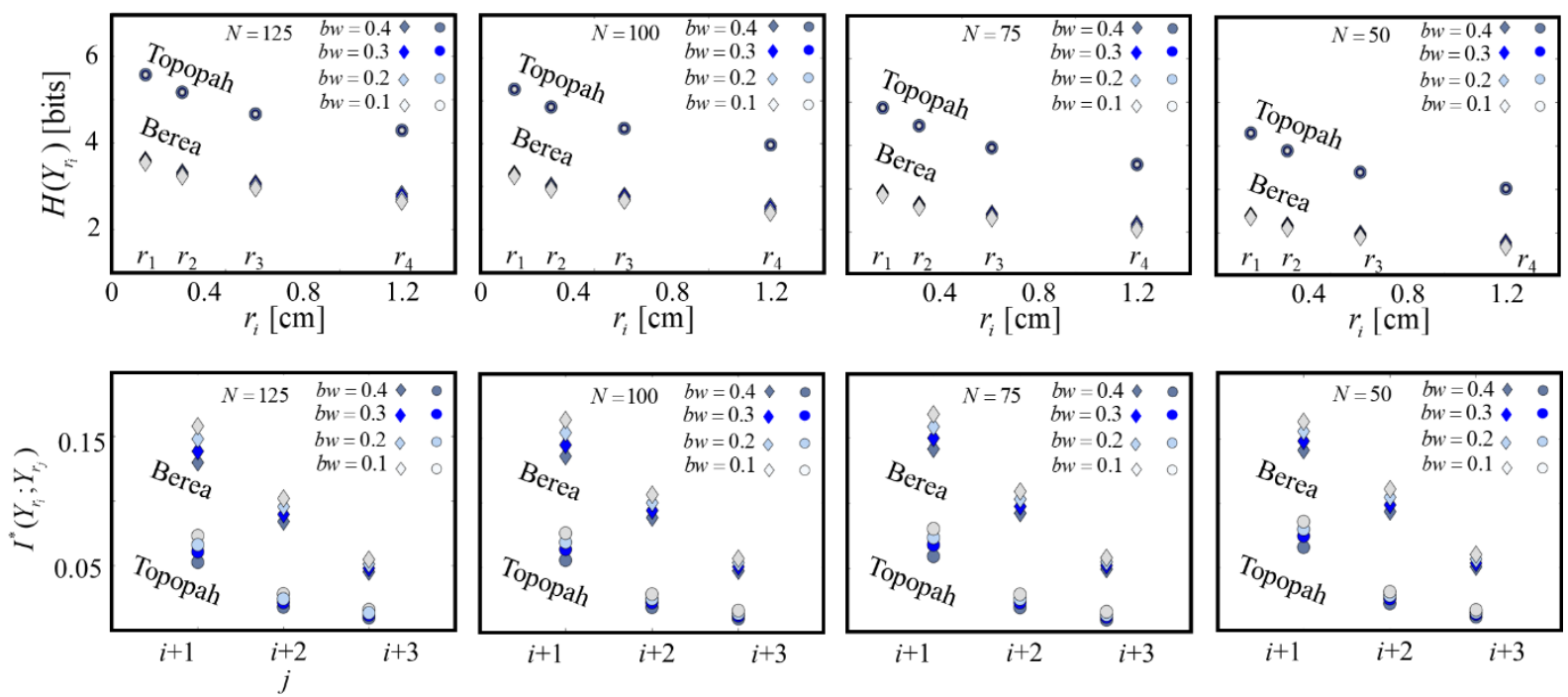

Figure S1. Shannon entropy ( $\left.H\left(Y_{r_{i}}\right)\right)$ versus $r_{i}$ for the Topopah (circles) and the Berea (diamonds) samples for various numbers of bins $(N=[50,75,100,125])$ and sizes of the kernel bandwidth ( $b w$ $=[0.1,0.2,0.3,0.4])$. Bivariate normalized mutual information $I^{*}\left(Y_{r_{j}} ; Y_{r_{1}}\right)=I\left(Y_{r_{j}} ; Y_{r_{1}}\right) / H\left(Y_{r_{1}}\right)$ between data at the support scale $r_{1}$ (i.e., $Y_{r_{1}}$ ) and data at larger support scales, considering the Berea and the Topopah rock samples for various numbers of bins $(N=[50,75,100,125])$ and sizes of the kernel bandwidth $(b w=[0.1,0.2,0.3,0.4])$. Similar results are obtained considering data at the support scale $r_{2}$ (i.e., $Y_{r_{2}}$ ) and data at larger support scales.

Inspection of Figure $\mathrm{S} 1$ suggests that the values of the Shannon entropy $\left(H\left(Y_{r_{i}}\right)\right)$ and of the normalized bivariate mutual Information $\left(I^{*}\left(Y_{r_{j}} ; Y_{r_{1}}\right)\right)$ slightly depend on the number of bins $(N)$ and on the size of the bandwidth $(b w)$ employed in the Kernel Density Estimator (KDE) procedure, while the overall patterns of $H\left(Y_{r_{i}}\right)$ and $I^{*}\left(Y_{r_{j}} ; Y_{r_{1}}\right)$ do not exhibit substantial variations with $N$ and $b w$. 

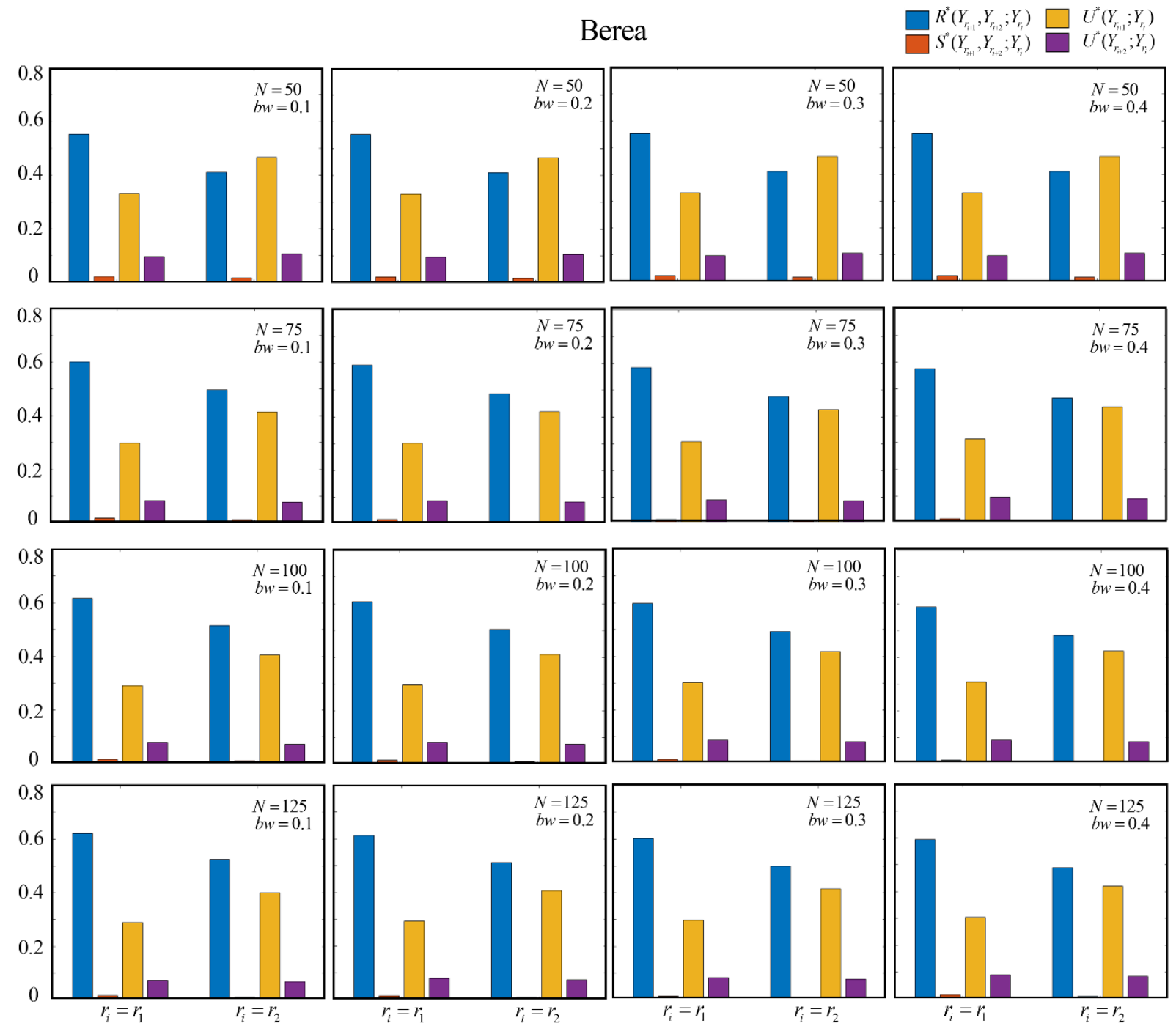

Figure S2. Information partitioning of the multivariate mutual information, $I\left(Y_{r_{i+1}}, Y_{r_{i+2}} ; Y_{r_{i}}\right)$, considering two triplets of data and $r_{i}=\left[r_{1}, r_{2}\right]$ for the Berea sample as a function of the number of bins $(N=[50,75,100,125])$ and the size of the kernel bandwidths $(b w=[0.1,0.2,0.3,0.4])$. For ease of comparison, we show the redundant, unique, and synergetic, contributions normalized by $I\left(Y_{r_{i+1}}, Y_{r_{i+2}} ; Y_{r_{i}}\right)$.

Inspection of Figure S2 suggests that the value of the information partitioning of the multivariate mutual information for the Berea sample slightly depends on the number of bins $(N)$ and on the size of the bandwidth $(b w)$ employed in the KDE procedure, while the overall patterns of the information partitioning for the Berea sample do not exhibit substantial variations with $N$ and $b w$. 

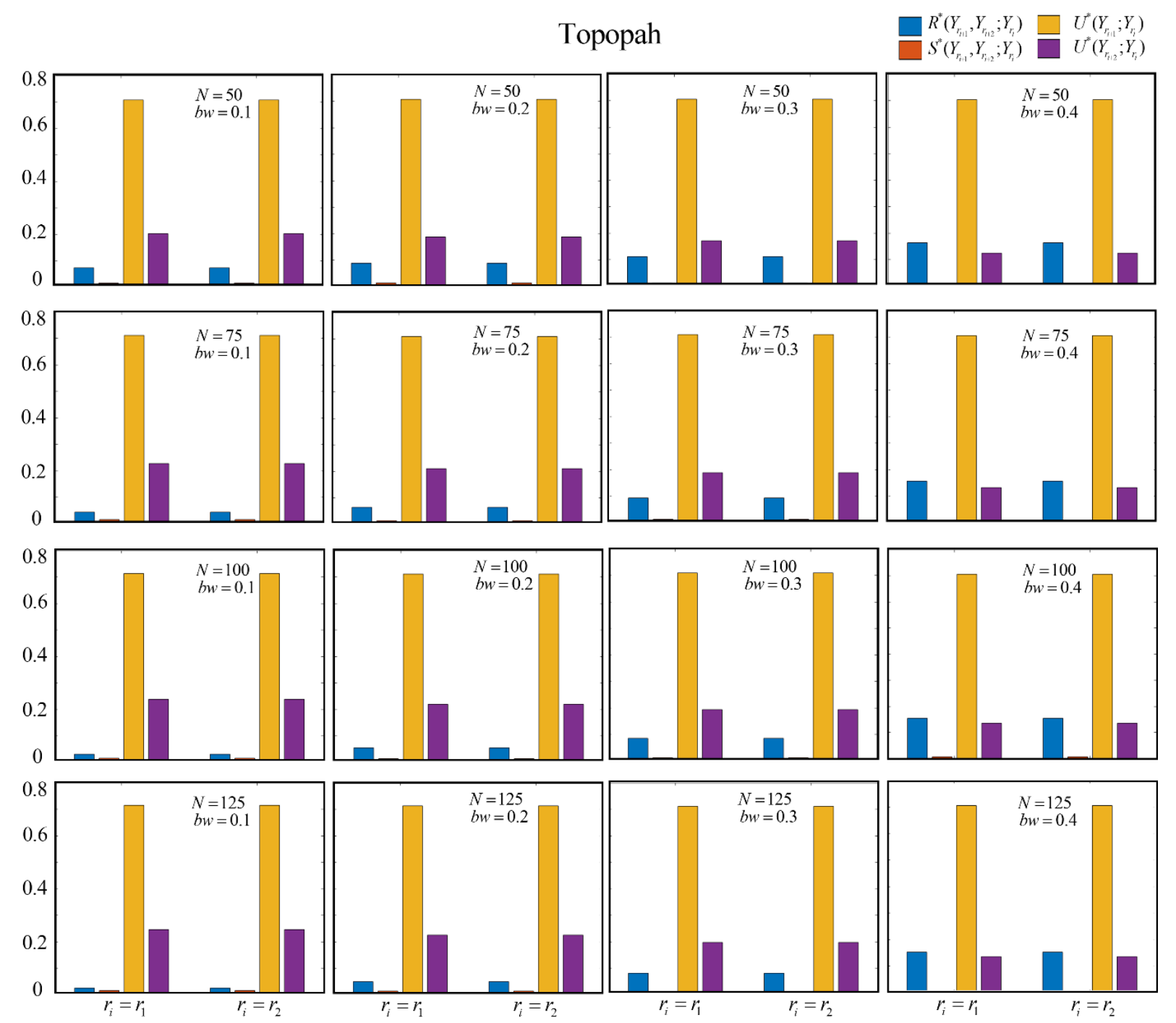

Figure S3. Information partitioning of the multivariate mutual information, $I\left(Y_{r_{i+1}}, Y_{r_{i+2}} ; Y_{r_{i}}\right)$, considering two triplets of data and $r_{i}=\left[r_{1}, r_{2}\right]$ for the Topopah sample as a function of the number of bins $(N=[50,75,100,125])$ and size of the kernel bandwidth $(b w=[0.1,0.2,0.3,0.4])$. For ease of comparison, we show the redundant, unique, and synergetic, contributions normalized by $I\left(Y_{r_{i+1}}, Y_{r_{i+2}} ; Y_{r_{i}}\right)$.

Inspection of Figure S3 suggests that the value of the information partitioning of the multivariate mutual information for the Topopah sample slightly depends on the number of bins $(N)$ and on the size of the bandwidth $(b w)$ employed in the KDE procedure, while the overall patterns of the information partitioning for the Berea sample do not exhibit substantial variations with $N$ and $b w$. 